\title{
Serum levels of Antioxidant in patients with Oral Squamous Cell Carcinoma: a preliminary study
}

\author{
Dr Neeta Bagul ${ }^{1}$, Dr Anjali Ganjre ${ }^{2}$, Dr Supriya Kheur ${ }^{3}$, Dr Deepali Patekar ${ }^{4}$, \\ Shreya Dasgupta ${ }^{5}$, Dr. Aditi Mahalle \\ ${ }^{I}$ Professor Department of Oral pathology and Microbiology,Dr. D.Y. Patil Deemed University, Dr.D.Y. Patil \\ Dental College and Hospital, Sant Tukaram Nagar, Pimpri, Pune 411018, India. \\ ${ }^{2}$ Senior Lecturer Department of Oral pathology and Microbiology Annasaheb Chudamani Patil dental college \\ Jawahar foundation, Sakri, Dhule, Maharashtra, India, 424002 \\ ${ }^{3}$ Professor Department of Oral pathology and Microbiology,Dr. D.Y. Patil Deemed University, Dr.D.Y. Patil \\ Dental College and Hospital, Sant Tukaram Nagar, Pimpri, Pune 411018, India. \\ ${ }^{4}$ M.D.S. Student (Part III) Departmnet of Oral pathology and Microbiology, Dr. D.Y. Patil Deemed University, \\ Dr.D.Y. Patil Dental College and Hospital, Sant Tukaram Nagar, Pimpri, Pune 411018, India. \\ ${ }^{5}$ B.D.S. Student (IV year) Dr. D.Y. Patil Deemed University, Dr.D.Y. Patil Dental College and Hospital, Sant \\ Tukaram Nagar, Pimpri, Pune 411018, India. \\ ${ }^{6}$ Senior Lecturer Department of Oral pathology and Microbiology, Dr. D.Y. Patil Deemed University, Dr. D.Y. \\ Patil Dental College and Hospital, Sant Tukaram Nagar, Pimpri, Pune 411018, India.
}

\begin{abstract}
Aim: To evaluate and compare superoxide dismutase and glutathione peroxidase in patients with oral squamous cell carcinoma with control subjects.

Material and Methods: A clinical study was conducted in which venous blood was collected from 25 histopathologically confirmed patients of oral squamous cell carcinoma and 25 control subjects and biochemically evaluated for levels of superoxide dismutase and glutathione peroxidase by Buetler method.

Results: Significantly increased levels of superoxide dismutase and glutathione peroxidase were observed in oral squamous cell carcinoma patients compared to control subjects. Using the Mann Whiteney U test, $p$ value of superoxide dismutase was found to be 0.026 and 0.017 of glutathione peroxidase which was statistically significant.

Conclusion: Endogenous antioxidant levels were found to be elevated in patients suffering from oral squamous cell carcinoma as compared to control subjects. The cause behind this may be increased oxidative stress and higher levels of circulating free radicals in patients with oral squamous cell carcinoma. The body's defense mechanisms would play an important role in the form of antioxidants and try to minimize the damage, adapting itself to the above stressful situation. Thus the increased activity of antioxidant enzyme may be a compensatory regulation in response to oxidative stress.
\end{abstract}

Keywords: antioxidants, glutathione peroxidase, oral squamous cell carcinoma, superoxide dismutase.

\section{Introduction}

Oral cancer is a public health threat, accounting for 274,300 new cases and 145,500 deaths worldwide in 2002, of which two-thirds took place in developing countries [1]. In India, it is the most common form of cancer and of cancer-related death in men (83,000 new cases and 46,000 deaths yearly) [1]. Though the role of tobacco and areca nut is well established with their etiopathogenesis and mutative changes in the oral epithelium, newer research indicates that body's own defence has a pivotal role to play in progression and prognosis of oral cancer. Substantial evidence has established the carcinogenic role of Reactive Oxygen Species (ROS) in the initiation and promotion of cancer [2] ROS is a molecule or molecule fragments containing unpaired electrons in the valence shell that is capable of existing independently (free) [3]. The ROS produced in cells include hydrogen peroxide, hypochlorous acid, and free radicals such as the hydroxyl radical and the superoxide anion. The hydroxyl radical is particularly unstable and will react rapidly and non-specifically with most biological molecules [4]. These species play a dual role as both toxic and beneficial compounds. At low or moderate levels, ROS and reactive nitrogen species (RNS) exert beneficial effects on cellular responses and immune function. At high concentrations, they generate oxidative stress, a deleterious process that can damage cell structures. The human body has several mechanisms to counteract oxidative stress by producing antioxidants, which are either naturally produced in situ, or externally supplied through foods and/or supplements. Endogenous and exogenous antioxidants act as "free radical scavengers" by preventing and repairing damages caused by ROS and RNS, and therefore can enhance the 
immune defense and lower the risk of cancer and degenerative diseases. The major antioxidant enzymes directly involved in the neutralization of ROS and RNS are: superoxide dismutase (SOD), catalase (CAT), glutathione peroxidase (GPx) and glutathione reductase (GRx) [5, 6].

The antioxidant enzymes SOD, CAT and GPx serve as the backbone of cellular antioxidant defense mechanism [7]. Increase in the level of SOD in blood of patients with oral squamous cell carcinoma (OSCC) which may be due to overwhelming free radicals due to increased levels of lipid peroxides circulating in the OSCC patients. Increased scavenging of lipid peroxides may have a significant role in carcinogenesis [8]. Studies have shown that levels of antioxidants are significantly increased in other malignancies $[9,10,11]$ as compared with control subjects. In contrast decreased antioxidant levels in patients with OSCC [12] and after radiotherapy treatment [13] were also reported.

Thus literature review revealed conflicting results as to whether antioxidant are increased or decreased in patients suffering from OSCC. We designed this study to evaluate the actual levels of antioxidants in the patients with OSCC with tobacco habit, reporting to our outpatient department. This is to eliminate the bias keeping in mind the increase in pharmaceutical prescription of antioxidants in OSCC patients.

\section{Material and methods}

2.1 Study design- Twenty five adult patients diagnosed histopathologically on incisional biopsy as OSCC in Department of Oral Pathology, Dr D.Y. Patil Dental College and Hospital at Sant Tukaram Nagar in western Maharashtra in India, were selected for the clinical study. An equal number of control subjects of similar age group were also chosen who were consuming tobacco for at least two years. All the subjects were explained about the objectives of the study. Written consent for willing to participate in the study was obtained. Ethical committee approval was taken for the study. The inclusion criteria's were a) age group between 35-65 years, b) patients giving history of tobacco abuse for at least two years, c)Patient giving history of present lesion (OSCC) to be less than 3 months. d) Patients agreeing for biopsy and blood examination. The exclusion criteria's were a) Patients not willing to participate in the study b) Patients suffering from systemic diseases or having clinical manifestations of tobacco abuse in form of potentially malignant diseases. Table 1 shows the characteristics of OSCC patients and control subjects.

Table 1- Characteristics of OSCC patients and control subjects.

\begin{tabular}{|l|l|l|}
\hline Total number of healthy subjects & Females & 10 \\
& Males & 15 \\
& Age range (years) & $35-65$ years \\
\hline Total number of OSCC patients & Females & 6 \\
& Males & 19 \\
& Age range (years) & $36-87$ years \\
\hline Site & Tongue & 2 \\
& Alveolar mucosa & 11 \\
& Retromolar region & 8 \\
& Cheek mucosa & 2 \\
& Floor of the mouth & 2 \\
\hline Histological grading & Well differentiated OSCC & 20 \\
& Moderately differentiated OSCC & 5 \\
\hline
\end{tabular}

Taking standardized aseptic precautions; blood samples (approximately $5 \mathrm{ml}$ ) were collected from anti cubital fossa. $2 \mathrm{ml}$ of whole blood was stored in ACD anticoagulant bulb at $7^{\circ} \mathrm{c}$ until further analysis, while $3 \mathrm{ml}$ of blood was centrifuged at $3000 \mathrm{rpm}$ for 10 minutes to obtain serum.

2.2 Biochemical Measurements- The estimation of GPx was done by the method of Beutler et al [14] Absorbance of final product which is formed was measured by Spectrometer manufactured by Elico Company, at $412 \mathrm{~nm}$.

Serum analysis for SOD was done by Marklund and Marklund method [15]. This method utilizes the inhibition of auto-oxidation of pyrogallol by SOD. The absorbance from the final product was continuously measured for 4 minutes at $420 \mathrm{~nm}$ at intervals of 30 seconds. One unit of SOD is described as the amount of enzyme required to cause $50 \%$ inhibition of pyrogallol auto-oxidation per $30 \mathrm{ml}$ of assay mixture and is measured as unit per $\mathrm{ml}$.

Levels of SOD and GPx were measured with the help of spectrophotometer. Data obtained was subjected to Kolmogorov - Smirnov test to check its normalcy of distribution. Since the data was not found to be normal Mann Whitney $-U$ test was used. $\mathrm{p}$ value $<0.05$ was considered for statistical significance. 


\section{Results-}

3.1 Table 2 - Descriptive statics of the study groups

\begin{tabular}{|l|l|l|l|l|l|}
\hline Groups & Antioxidant levels & N & Mean & Std. Deviation & Std. Error Mean \\
\hline OSCC & SOD levels (SOD1) & 25 & 3.92 & 1.75 & \\
& GPx levels (GPX1) & 25 & 0.03 & 0.02 & 0.35 \\
& SOD levels (SOD) & 25 & 3.11 & 1.95 & 0.00 \\
Control & GPx levels (GPX) & 25 & 0.02 & 0.02 & 0.39 \\
\hline
\end{tabular}

Table 2 depicts the descriptive statics of the various study groups. The mean value of SOD in OSCC patients is $3.92{ }_{ \pm} 1.75$ whereas in normal individual it is found to be $3.11_{ \pm} 1.95$. The mean of GPx in OSCC patients is 0.03 ${ }_{ \pm} 0.02$ and $0.02{ }_{ \pm} 0.02$ in normal individual.

3.2 Table 3-Comparison of serum levels of SOD and GPx in study and control group patients using the Mann Whitney U test.

\begin{tabular}{|c|c|c|c|c|}
\hline Variable & Group & Mean rank & Mann-whitney u & $\begin{array}{l}\text { Significance } \\
\text { p value }\end{array}$ \\
\hline \multirow{2}{*}{ SOD } & Normal & 20.90 & \multirow{2}{*}{197.50} & \multirow{2}{*}{$.026^{*}$} \\
\hline & Study & 30.10 & & \\
\hline \multirow{2}{*}{ GPx } & Normal & 20.60 & \multirow{2}{*}{190.00} & \multirow{2}{*}{$.017^{*}$} \\
\hline & Study & 30.40 & & \\
\hline
\end{tabular}

*p value $<0.05$ was considered statistically significant.

Table 3 shows that the difference between the antioxidant levels of the two groups is statistically significant with the antioxidants being more in the OSCC patients as compared to the normal individuals.

\section{Discussion}

Oral cavity cancer is one of the ten most frequent cancers in the world. Cancer is the product of interaction of genetic factors and environment exposures. Abuse of alcohol and tobacco has serious nutritional implications for the host and generates increased production of reactive free radicals. Active oxygen species and other reactive free radicals can mediate phenotype and genotype alterations causing mutations. Antioxidants or free radical scavengers, both natural and synthetic, neutralize metabolic products, interfere with activation of pro-carcinogens, prevent binding of carcinogens to DNA, inhibit chromosome aberrations, restrain replication of the transformer cells, and suppress action of pre-cancerous lesions such as leukoplakia and erythroplakia [16].

The common antioxidants are SOD and GPx. SOD's are a class of closely related enzymes that catalyse the breakdown of the superoxide anion into oxygen and hydrogen peroxide. SOD enzymes are present in almost all aerobic cells and in extracellular fluids [6]. The glutathione system includes glutathione reductase, glutathione peroxidases and glutathione S-transferases. By products of lipid peroxidation causes marked alteration in the structural integrity and function of cell membranes. GPx is an enzyme containing four selenium co-factors that catalyses the breakdown of hydrogen peroxide and organic hydropeoxides [17]. GPx is the most abundant and is a very efficient scavenger of hydrogen peroxide, while glutathione pro-oxidase is the most active lipid hydroperoxides [18].

Thus to study the correlation between levels of antioxidants in patients suffering from OSCC, serum levels of SOD and GPx which are the major enzymatic antioxidant defense system responsible for scavenging free radicals and nascent oxygen were estimated in normal individuals and patients with OSCC. Results showed a statistically significant ( $\mathrm{p}$ value $<0.05$ ) increase in the levels of GPx and SOD in OSCC patients as compared with control subjects.

The possible explanation for our results would be that patients who are in the initial stages of OSCC have high oxidative stress and lipid peroxidation. Also the level of free radicals in their body may have increased and as a compensatory mechanism body tries to increase the levels of antioxidants. Hence the increased serum levels of antioxidants show that there is a natural defense mechanism followed by the body to combat carcinogenesis. Our results are similar to Gokul et al., who found an increase in the level of SOD in blood of patients with OSCC. They explained this to the overwhelming free radicals due to increased levels of lipid peroxides circulation in the OSCC patients. Thus increased scavenging of lipid peroxides may have a significant role in carcinogenesis[8]

Oxidative environment in newly established malignant phenotype may provoke oxidants to activate gene expression through the antioxidant responsive elements. It is supposed that overexpression of endogenous 
antioxidants are important components of antioxidative defense through the process of malignant transformation .These facts might explain strongly enhanced SOD activity at lower stage of malignant disease that they found in two patients at clinical stage I. [9] The results are in contrast to Khanna R, S. Syed Sultan Beevi who has stated that the levels of antioxidants are considerably decreased in cancer patients than normal individuals $[17,19]$. Patel $\mathbf{J}$ et al., found erythrocyte SOD activities to be significantly increased in OSCC than normal individuals with smoking habits and in patients with pre-cancerous lesions [2]. Gurudath $\mathrm{S}$ et al also found that Erythrocyte -SOD (E-SOD) and GPx levels were decreased in not only OSCC but oral leukoplakia and oral submucous fibrosis also [20]. Kumar Chandan Srivatsav et al found decreased levels of antioxidants with a corresponding increased level of free radicals in patients with OSCC when compared with control subjects [21]. Raghvendra $U$ et al found an increased SOD activity in smokers and explained it to be an adaptive mechanism of the body to counter act the oxidant factor as they found an increased level of oxidative stress. But, they further noted decreased levels of SOD in cases of OSCC patients. They thought it to be due to higher magnitude of oxidative stress since all their patients were in advanced clinical stages with a large tumor burden [22]. It might be so that once chemotherapy, radiation or surgery treatment starts for cancer patients the body no longer is the primary and sole defender against the free radicals. Hence the levels of antioxidants may decrease since various other methods are being used simultaneously to stop neoplasm. In our study, most of the patients were from low socioeconomic strata with not a very rich nutritious diet. Still these patients exhibited an increase in antioxidant levels. Probably, our body is capable of producing antioxidants to combat the oxidative injury, at least in initial stages. Thus the above study might be useful in opening doors to previous questions on the levels of antioxidants in cancer patients. The standard protocol of administration of antioxidant to the patient suffering from OSCC becomes questionable, as our study shows increase in endogeneous antioxidants.

There are several limitations to the study, primarily low sample size. But it can be marked as a pilot study, thereby improving our understanding of cancer progression and the true role of antioxidants in cancer therapy.

\section{Conclusion-}

In the present study, the level of antioxidant in serum i.e. SOD and GPx were shown to be increased in OSCC as compared to control subjects with tobacco habit but exhibiting no clinical manifestations. This could be because of body's defense mechanism. In initial or early stages these antioxidant levels increases thereby showing an evidence of endogenous activity. As our study is of preliminary nature ,thus , larger sample size and multi institutional study could further support our hypothesis stating the auto regulation of antioxidant system in OSCC.

\section{Acknowledgment}

This study was approved as a short term research by Indian Council of Medical Research (ICMR) and funded by the same.

\section{References}

[1]. Sankaranarayanan R, Ramadas K, Thomas G, Muwonge R, Thara S, Mathew B et al .Effect of screening on oral cancer mortality in Kerala, India: a cluster-randomised controlled trial. Lancet, 365(1495), 2005, 1927-1933.

[2]. Patel JB, Shah FD, Shukla SN, Shah PM, Patel PS. Role of nitric oxide and antioxidant enzymes in the pathogenesis of oral cancer. Journal of cancer research and therapeutics, 5(4), 2009, 247-253.

[3]. Fiaschi AI, Cozzolino A, Ruggiero G, Giorgi G. Glutathione, ascorbic acid and antioxidant enzymes in the tumor tissue and blood of patients with oral squamous cell carcinoma. European review for medical and pharmacological sciences, 9(6), 2005, 361-67.

[4]. Yu BP. Cellular defenses against damage from reactive oxygen species. Physiological Reviews, 74(1), 1994, 139-162.

[5]. Gonzales R, Auclair C, Voisin E, Gautero H, Dhermy D, Boivin P. Superoxide dismutase, catalase, and glutathione peroxidase in red blood cells from patients with malignant diseases. Cancer Research, 44 (9), 1984, 4137-4139.

[6]. Pham-Huy LA, He H, Pham-Huy C. Free radicals, antioxidants in disease and health. International Journal of Biomedical Science, 4(2), 2008, 89-96.

[7]. Manoharan S, Kolanjiappan K, Suresh K, Panjamurthy K. Lipid peroxidation \&amp; antioxidants status in patients with oral squamous cell carcinoma. The Indian journal of medical research, 122, (6), 2005, 529-534.

[8]. Gokul S, Patil VS, Jailkhani R, Hallikeri K, Kattappagari KK. Oxidant-antioxidant status in blood and tumor tissue of oral squamous cell carcinoma patients. Oral Diseases, 16(1), 2010, 29-33.

[9]. Suprapaneni KM, Venkata Ramana G. Lipid peroxidation and antioxidant status in patients with carcinoma of prostrate. Indian Journal of Physiology and Pharmacology, 2006, 50 (4), 350-354.

[10]. Bogdanovic V, Tursijan S, Dordevic M, Nikolic A, Mrdanovic J, Jakimov D. et al. Activity of lactate dehydrogenase and superoxide dismutase in the circulation of patients with breast carcinoma. Arch Oncol, 16 (3-4), 2008, 39-41.

[11]. Pejić S, Todorović A, Stojiljković V, Kasapović J Pajović SB. Antioxidant enzymes and lipid peroxidation in endometrium of patients with polyps, myoma, hyperplasia and adenocarcinoma. Reproductive Biology and Endocrinology, 2009, 7, 49. Open access.

[12]. Yang J, Lam EW, Hammad HM, Oberley TD, Oberley LW. Antioxidant enzyme levels in oral squamous cell carcinoma and normal human oral epithelium. Journal of oral pathology and medicine, 31(2), 2002, 71-77.

[13]. Sabitha KE, Shyamaladevi CS. Oxidant and antioxidant activity changes in patients with oral cancer and treated with radiotherapy. Oral Oncology, 35(3), 1999, 273-277.

[14]. Beutler E. The hemolytic effect of primaquine and related compounds: a review. Blood, 14(2), 1959, 103-139. 
[15]. Marklund SL. Clinical aspects of superoxide dismutase. Medical Biology, 62(2), 1984, 130-134.

[16]. Beenadas. Antioxidants in the treatment and prevention of oral cancer. Kerala Dental Journal, 31 (4), 2008, 24-33.

[17]. Khanna SS, Karjodkar FR. Circulating immune complexes and trace elements (Copper, Iron and Selenium) as markers in oral precancer and cancer: a randomised, controlled clinical trial. Head \& face medicine, 2(33), 2006, 1-10.

[18]. Bathi RJ, Rao R, Mutalik S.GST null genotype and antioxidants: risk indicators for oral pre-cancer and cancer. Indian journal of dental research, 20(3), 2009, 298-303.

[19]. Beevi SS, Rasheed AM, Geetha A. Evaluation of oxidative stress and nitric oxide levels in patients with oral cavity cancer. Japanese journal of clinical oncology, 34(7), 2004, 379-85.

[20]. Gurudath S, Naik RM, Ganapathy KS, Guruprasad Y, Sujatha D, Pai A. Superoxide dismutase and glutathione peroxidase in oral submucous fibrosis, oral koplakia and oral cancer: A comparative study. Journal of Orofacial Science, 4(2), 2012, 114-119.

[21]. Srivastava KC, Austin RD, Srivastava D, Sethupathy S, Rajesh S. A case control study to evaluate oxidative stress in plasma samples of oral malignancy. Contemporary Clinical Dentistry. 3(4), 2012, 271-276.

[22]. Raghavendra U, D'Souza V, D'Souza B. Erythrocyte malondialdehyde and antioxidant status in oral squamous cell carcinoma patients and tobacoo chewers/smokers. Biomed Research.21 (4), 2010, 441-444. 